\title{
Conditional reference charts to assess weight gain in British infants
}

\author{
T J Cole
}

\begin{abstract}
Growth monitoring in infancy is a useful tool for detecting growth disorders and failure to thrive. However, current weight charts do not monitor growth as such, they only identify infants whose weight centile is low and/or falling. A reference of conditional weight gain is described which compares an infant's current weight with that predicted from their previous weight, allowing for the fact that on average, light infants tend to grow faster than heavier infants. The reference, which expresses conditional weight gain as an SD score or centile, is based on the UK 1990 weight reference supplemented with correlation data on 223 infants from the Cambridge Infant Growth Study measured regularly between 4 weeks and 2 years of age. The reference is validated with data on 727 infants from the Newcastle Regional Health Authority database. The conditional reference provides a valid assessment of the weight gain of British infants, over time periods of four or more weeks, throughout the first two years of life.
\end{abstract}

(Arch Dis Child 1995; 73: 8-16)

Keywords: growth monitoring, weight gain, conditional reference, velocity.

How fast does an infant grow? This apparently innocuous question still awaits a complete answer, despite the substantial literature that exists on the subject. It is an important question, as it affects the construction of reference charts to monitor growth in infancy.

Charts of weight attained, also known as weight distance charts, are derived from cross sectional data, and they allow weight to be expressed as a centile relative to the reference population, adjusted for age and sex. Such charts are also used to monitor weight velocity, on the grounds that a normally growing infant stays close to his or her chosen weight centile. This means that weight faltering is often inferred from the infant's weight falling across centiles.

Weight distance centiles should not be used in this way, however, as they are derived from cross sectional data and cannot quantify changes in weight. What is needed is a velocity chart, based on longitudinal data, consisting at its simplest of mean and SD of weight increments between specified ages. ${ }^{1-3}$ The mean and SD both change with age, but to complicate matters the SD also depends on the time between measurements - the measurement error is greater over shorter periods of time. ${ }^{4} \mathrm{~A}$ saltatory pattern of growth ${ }^{5}$ will also tend to increase variability on a short timescale. This is why infant velocity charts are based on a fixed time interval, for example one month or three months, between measurements. However, infants are not usually measured this way, but are seen at unstructured times. Unless the measurement times can be fixed in advance, for example in a longitudinal research study, velocity charts are of limited value.

An alternative to the velocity chart is the Sheffield weight chart, ${ }^{6}$ which is a distance chart modified to interpret centile crossing. The usual 3rd, 50th, and 97th distance centile are augmented with extra channel lines, which are spaced to ensure that just $5 \%$ of infants shift up or down by one channel width or more during a two week interval. By good fortune, over an eight week period, the same percentage of infants can be expected to shift up or down by two channel widths.

The Sheffield chart provides velocity information on a distance chart, but like the velocity chart it has deficiencies. The first is that for measurements appreciably more than eight weeks apart, the channel widths are uninformative. The second and more fundamental issue is that of regression to the mean. Over a period of time, infant weight tends to drift (or regress) towards the median - the tendency is to become less extreme with passing time. Thus an infant on say the 2 nd weight centile is likely to show catch-up growth, whereas 98th centile infants tend on average to catch down. ${ }^{7}$

A weight velocity reference that compensates for regression to the mean is called a conditional reference.$^{8-10}$ It answers the question: 'Knowing the infant's previous weight, what is her likely weight now?' Wright et al have recently used this concept to derive the thrive index, a measure of change in weight SD score between 6 weeks and 12 months which takes into account the differential patterns of growth seen in small and large babies. ${ }^{7}$ Infants whose thrive index is below the cut off are referred for further investigation.

The aim of this paper is to describe a conditional reference for infant weight gain which 
generalises the approach of Wright et $a l^{7}$ by covering any pair of ages between 4 weeks and 2 years. It calculates weight gain in terms of the change in weight distance SD score based on the UK 1990 reference, ${ }^{11}$ adjusted for regression to the mean, and gives the result as a SD score for weight gain.

\section{Methods}

REGRESSION TO THE MEAN

The concept of regression to the mean is central to the paper, yet it is unfamiliar and deserves explanation. Fundamentally a statistical phenomenon, it states that if individuals or groups of individuals are weighed once, and later weighed again, their weight centile on the second occasion tends, on average, to be nearer the median than on the first occasion. This may seem counter intuitive - surely an infant on say the 2 nd weight centile ought on average to stay there rather than move to a higher centile?

It is important to realise this is a phenomenon about averages - it does not say that every infant on the 2 nd centile will catch up, only that a majority will. To see why, consider a randomly selected child at say 12 months of age. Knowing nothing about her we expect her to be average for her age, with an expected weight on the population median. Now imagine we are given the information that her weight at 9 months was on the 2 nd centile. How should this extra knowledge alter our expectation of her weight at 12 months? The fact that she was below the median three months earlier obviously means that she ought still to be below the median, but by how much?

There is a range of possibilities. At one extreme, if weight tracks perfectly between 9 and 12 months, then we should expect her to remain on the 2 nd centile. Conversely, if there is no tracking at all between the two ages, our initial expectation will be unaltered, and we should still expect her to be on the median at 12 months. Thus the alternatives range from the 2 nd to the 50th centile, depending on how strongly weight tracks between 9 and 12 months. (Her actual centile at 12 months may well be below the 2nd centile, but her average or expected centile will be above it.)

In this context tracking is synonymous with correlation, and perfect tracking requires a perfect correlation. This is impossible, so the extreme case of expecting her to remain on the 2 nd centile is ruled out. Her predicted weight centile at 12 months has to be above the 2 nd centile - less extreme than her weight centile at 9 months - so her weight appears to regress towards the median. The same argument, in the reverse direction, applies to infants with weight centiles above the median.

The amount of regression to the mean depends on how highly correlated weights are at the two ages, so the way to adjust for regression to the mean is to quantify this correlation.
SUBJECTS AND DATA

The Cambridge Infant Growth Study was set up in 1983 to monitor the pattern of growth of infants being fed according to Department of Health dietary guidelines. ${ }^{12}$ Two hundred and fifty four mothers from the City of Cambridge were recruited during pregnancy to take part, in four separate cohorts. Their infants were weighed and measured every four weeks $( \pm 3$ days) from 4 to 52 weeks, and at 18 and 24 months. They were all at least 35 weeks' gestation, and no adjustment has been made here for gestational age. At each visit, weight and five other anthropometric measures were obtained using standard methods. ${ }^{12}$ Thus each infant was weighed up to 15 times between the ages of 4 weeks and 24 months. Two hundred and twenty three infants (114 boys, 109 girls) ( $87 \%$ of those recruited) were seen on all 15 occasions. The second and later cohorts $(n=183)$ were also seen at 15 months of age, and their data have been included in the validation process.

A separate dataset of weights was obtained from the Newcastle region child health computer (courtesy of Dr Charlotte Wright) to validate the conditional weight gain reference. The dataset included 4879 weights from 761 infants, 727 seen twice or more, weighed whenever the infant was brought to the clinic between the ages of 4 weeks and 24 months. The median number of visits per infant was seven, maximum 10 .

\section{NOTATION}

Traditionally, weight and weight change are described in terms of the child's journey to adulthood, so that attained weight at a particular age is called weight distance, while the rate of weight gain between two ages is called weight velocity. Weight velocity is actually the slope of the line joining two weights on the weight distance chart.

However, once regression to the mean is acknowledged, the concept of velocity becomes unhelpful. It implies that all children of a particular age have the same expected velocity, which is not the case - light children have a higher expected velocity than heavy children. To avoid possible confusion the term velocity, or rate of weight gain, is avoided in this paper and gain is used instead. The definition of weight gain does not adjust explicitly for the time interval between measurements. Instead it is expressed as an SD score, called SDS $_{\text {gain }}$, which summarises how the child's weight gain over a period of time compares with the gain expected in an average child of the same initial weight, measured at the same two ages. Weight distance is also expressed as an SD score, usually with the suffix 1 or 2 to indicate the measurement occasion.

\section{STATISTICAL ANALYSIS}

All the Cambridge and Newcastle weights were converted to $\mathrm{SD}$ scores using the UK 1990 weight reference. ${ }^{11}$ This adjusts the 
weight distribution for skewness, and allows weight distance to be converted to an exact SD score (see Appendix). The Cambridge data were used with other datasets in the construction of the UK reference, adjusted upwards to match the other datasets, and so should be expected to fit the reference well.

The conditional weight gain reference compares an infant's current weight distance SD score with the value predicted from their weight SD score on the previous measurement occasion. This is obtained from the linear regression of current weight $\mathrm{SD}$ score (call it $\mathrm{SDS}_{2}$ ) on previous weight SD score $\left(\mathrm{SDS}_{1}\right)$. It can be shown that the predicted value of $\mathrm{SDS}_{2}$ is given by r.SDS ${ }_{1}$, where $r$ is the correlation coefficient between $\operatorname{SDS}_{1}$ and $\operatorname{SDS}_{2}, 4713$ and that it is normally distributed with $\mathrm{SD} \sqrt{1-\mathrm{r}^{2}}$. The correlation coefficient is always less than one, so that $\mathrm{SDS}_{2}$ is expected to become smaller (that is nearer to zero, the median) with passing time, and the correlation is a direct measure of regression to the mean. The absence of an intercept in the regression equation, the fact that the regression coefficient is equal to the correlation, and the form of the $S D$, all arise from the dependent and independent variables being SD scores. ${ }^{4}$

Both the mean and SD of $\mathrm{SDS}_{2}$ conditional on $\mathrm{SDS}_{1}$ are known, so the conditional gain can itself be expressed as an SD score. The general formula to express quantity $\mathrm{X}$ as an $\mathrm{SD}$ score is:

$$
\text { SD score }=\frac{X-\operatorname{mean}(X)}{\operatorname{SD}(X)} \text {. }
$$

Here $\mathrm{X}$ is ' $\mathrm{SDS}_{2}$ conditional on $\mathrm{SDS}_{1}$ ', which gives the conditional gain $\mathrm{SD}$ score:

$$
\mathrm{SDS}_{\mathrm{gain}}=\frac{\mathrm{SDS}_{2}-\mathrm{r} \cdot \mathrm{SDS}_{1}}{\sqrt{1-\mathrm{r}^{2}}}
$$

Equation (1) is a SD score for conditional weight gain, and can be expressed as a centile. The correlation coefficient $r$ between SDS $_{1}$ and $\mathrm{SDS}_{2}$ in the equation varies according to the two ages of measurement, and can be obtained from the Cambridge data for any pair of ages when the infants were measured. There were 15 measurement ages, providing 105 distinct age pairs.

\section{MODELLING CORRELATION}

However, for any ages intermediate to the measurement ages, the correlations cannot be obtained - they have to be interpolated from the known correlations. This is done using a form of regression analysis, where the correlation is modelled as a function of the two ages of measurement. In the analysis, each pair of ages is converted to its mean on the one hand, and the time gap between ages on the other. So for example four week and eight week measurements are treated as a mean age of 6 weeks and a time gap of four weeks. Overall the mean age varies between 6 weeks and 21 months, and the time gap ranges from four weeks to 23 months.
Each of the 105 calculated correlations $r$ is transformed using Fisher's transformation ${ }^{14}$ :

$$
Z=\frac{1}{2} \log _{e}\left[\frac{1+r}{1-r}\right]
$$

This makes all the correlation variances equal to $1 /(n-3)$, where $n$ is the sample size used to calculate the correlation. The transformed correlation is then modelled as the dependent variable in a multiple regression equation involving sex, powers of the mean age and time gap, and interactions between them. ${ }^{15}$ The value of $\mathrm{Z}$ predicted from the regression equation for a particular pair of ages can then be converted back to a correlation using the formula:

$$
\mathrm{r}=\frac{\exp (2 \mathrm{Z})-1}{\exp (2 \mathrm{Z})+1}
$$

and substituted into equation (1).

To validate the method, pairs of weights for both Cambridge and Newcastle infants are converted to $\mathrm{SDS}_{\text {gain }}$ using equation (1). If the equation is appropriate, $\operatorname{SDS}_{\text {gain }}$ should have a mean of 0 , an SD of 1 , and it should be uncorrelated with the initial weight $\operatorname{SDS}_{1}$. These assumptions are tested by grouping the weight pairs according to their mean age and time gap, and summarising the mean and SD of $\mathrm{SDS}_{\text {gain }}$ and its correlation with $\mathrm{SDS}_{1}$ across the groups.

\section{Results}

Figure 1 shows the UK 1990 weight distance chart for boys in the first two years of life, with the seven centiles $2,9,25,50,75,91$, and 98 ; they correspond to SD scores from -2 to +2 spaced two thirds of an SD score apart. ${ }^{16}$ Details of how the centile curves are derived are given in the Appendix.

Table 1 gives the correlation matrix for weight distance SD score at each of the 15 measurement ages from 4 weeks to 24 months, based on 223 Cambridge boys and girls. The two sexes are not significantly different, so they

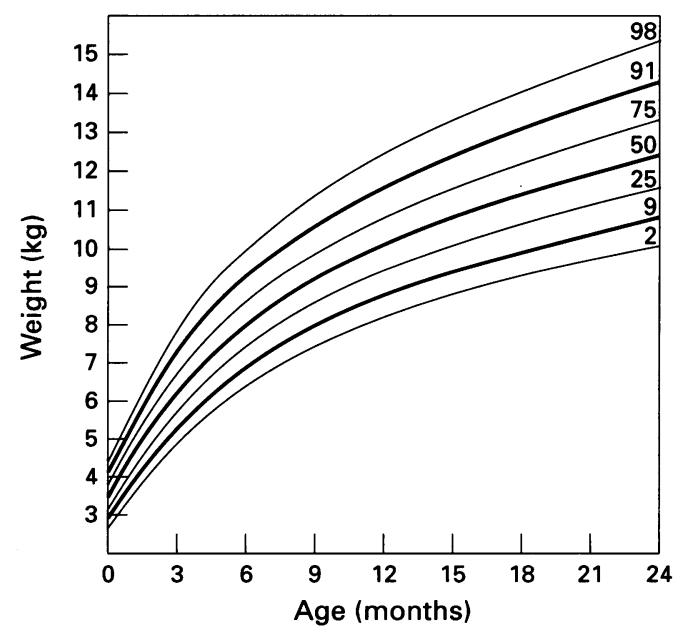

Figure 1 The UK 1990 boys weight distance chart from birth to 2 years, showing the 2nd, 9th, 25th, 50th, 75th, 91st, and 98 th centiles, spaced two thirds of an $S D$ score apart. 
Table 1 Correlation matrix for weight SD score at 15 ages during early childhood, based on data for 223 boys and girls seen on all 15 occasions

\begin{tabular}{|c|c|c|c|c|c|c|c|c|c|c|c|c|c|c|c|}
\hline \multicolumn{16}{|l|}{ Week } \\
\hline $\begin{array}{l}4 \\
8\end{array}$ & $\begin{array}{l}1 \\
0.911\end{array}$ & 1 & & & & & & & & & & & & & \\
\hline 12 & 0.823 & 0.945 & 1 & & & & & & & & & & & & \\
\hline 16 & 0.752 & 0.881 & 0.958 & 1 & & & & & & & & & & & \\
\hline 20 & 0.659 & 0.788 & 0.892 & 0.959 & 1 & & & & & & & & & & \\
\hline 24 & 0.611 & 0.738 & 0.845 & 0.918 & 0.967 & 1 & & & & & & & & & \\
\hline 28 & 0.588 & 0.705 & 0.811 & 0.891 & 0.945 & 0.971 & 1 & & & & & & & & \\
\hline 32 & 0.565 & 0.680 & 0.779 & 0.859 & 0.915 & 0.947 & 0.971 & 1 & & & & & & & \\
\hline 36 & 0.535 & 0.651 & 0.745 & 0.830 & 0.880 & 0.919 & 0.950 & 0.981 & 1 & & & & & & \\
\hline 40 & 0.504 & 0.624 & 0.718 & 0.800 & 0.850 & 0.893 & 0.924 & 0.961 & 0.979 & 1 & & & & & \\
\hline 44 & 0.488 & 0.599 & 0.687 & 0.771 & 0.823 & 0.867 & 0.901 & 0.940 & 0.959 & 0.975 & & & & & \\
\hline 48 & 0.474 & 0.587 & 0.673 & 0.755 & 0.809 & 0.851 & 0.890 & 0.925 & 0.949 & 0.963 & 0.977 & & & & \\
\hline 52 & 0.467 & 0.572 & 0.659 & 0.740 & 0.792 & 0.830 & 0.871 & 0.906 & 0.927 & 0.946 & 0.965 & 0.973 & & & \\
\hline 78 & 0.464 & 0.544 & 0.609 & 0.671 & 0.713 & 0.737 & 0.771 & 0.802 & 0.820 & 0.849 & 0.877 & 0.890 & 0.910 & & \\
\hline 104 & 0.483 & 0.584 & 0.634 & 0.680 & 0.706 & 0.727 & 0.746 & 0.764 & 0.775 & 0.804 & 0.822 & 0.826 & 0.850 & 0.929 & 1 \\
\hline Week & & & & & & & & & & & & & & & 104 \\
\hline
\end{tabular}

are combined. Generally speaking, the smaller the time gap between measurements and the greater the mean age, the higher the correlation.

The correlations immediately below the diagonal in table 1 measure regression to the mean from one measurement age to the next. For example between 4 and 8 weeks the correlation is 0.911 . A typical child with weight distance $\operatorname{SDS}_{1}=-2$ at 4 weeks regresses to the mean by this amount, so the expected distance $\mathrm{SDS}_{2}$ at 8 weeks is $-2 \times 0.911=-1.82$ units. Equally, between 8 and 12 weeks where the correlation is 0.945 , the same typical child is expected to move from $\mathrm{SDS}_{1}=-1.82$ to $\mathrm{SDS}_{2}=-1 \cdot 82 \times 0.945=-1 \cdot 72$.

Note that regression to the mean from 4 to 12 weeks is given by the $4-8$ and $8-12$ week correlations multiplied together, that is $-1.72=-2 \times 0.945 \times 0.911$. In general the amount of regression to the mean over a series of measurements every four weeks is obtained by multiplying together the correlations between adjacent measurements. So for measurements every four weeks from 4 to 52 weeks, regression to the mean can be obtained by multiplying together the 12 correlations below the diagonal in table 1 , which gives the result $0 \cdot 64$. So an average infant with weight $\mathrm{SDS}_{1}-2$ at 4 weeks will end up with $\mathrm{SDS}_{2}$

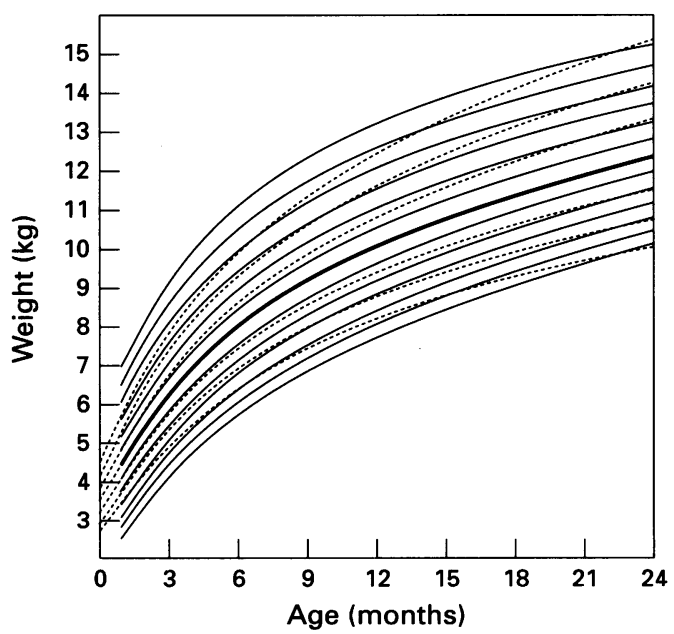

Figure 2 Boys conditional weight chart, showing the expected pattern of weight gain in infants measured every four weeks. The 13 solid lines represent infants with weight $S D$ scores at 4 weeks of age ranging between -4 and +4 , spaced two thirds of an SD score apart. Subsequently, as each infant grows, the SD score shrinks towards the median, as compared with the distance centiles of fig 1 (shown dotted). See text for details. near $-2 \times 0.64=-1.28$ at 52 weeks, on the 10 th centile, which corresponds to a whole channel shift upwards. By symmetry, relatively heavy infants starting at $\operatorname{SDS}_{1}+2$ tend to shift down to the same extent.

\section{MEDIAN CONDITIONAL WEIGHT}

Imagine now a set of 13 hypothetical infants, whose weight distance SD score at 4 weeks ranges from -4 to +4 in intervals of 0.67 , extending the SD score values used in fig 1.16 For each infant, predict their SD score at the end of successive four week measurement intervals using the correlations in table 1 , and then convert the resulting SD score back to weight. This gives a series of weights for each infant that defines the pattern of weight gain expected for infants starting at that particular SDS.

Figure 2 shows the result for boys, superimposed on the distance weight chart of fig 1 , and fig 3 is the corresponding chart for girls. The dashed curves are the seven distance centiles, and the solid curves the 13 conditional centiles. Each conditional centile represents the median pattern of growth of a child starting at that centile position and measured every four weeks. At four weeks the distance centiles and the central seven conditional centiles coincide, but thereafter the conditional centiles regress towards the median. At 6

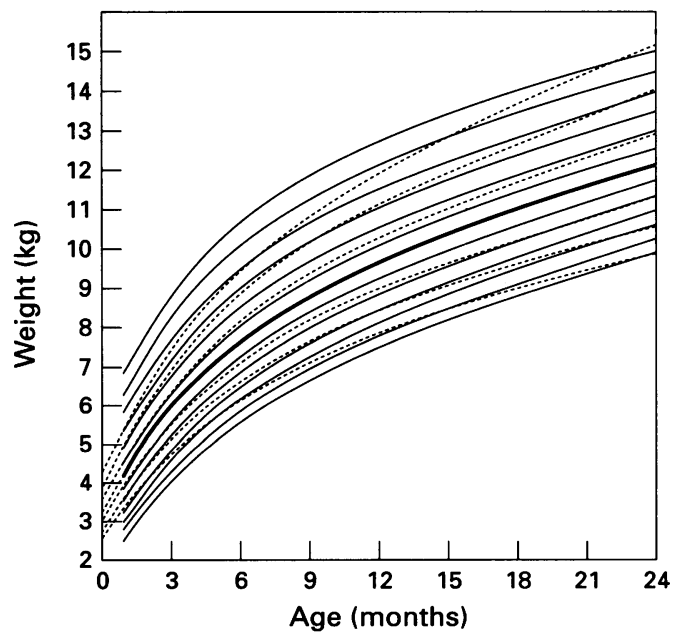

Figure 3 Girls conditional weight chart, showing the expected pattern of weight gain in infants measured every four weeks. See caption to fig 2 for details. 
Table 2 Calculation of conditional weight gain for infants measured at 4 and 52 weeks, who fall $2 S D$ score units during the interval. Effect of the starting SD score on the conditional gain $S D$ score and conditional gain centile

\begin{tabular}{|c|c|c|c|}
\hline \multicolumn{2}{|c|}{ Weight distance } & \multicolumn{2}{|c|}{$\begin{array}{l}\text { Conditional weight } \\
\text { gain from } 4-52 \text { weeks }\end{array}$} \\
\hline $\begin{array}{l}S D S_{1} \text { at } \\
4 \text { weeks }\end{array}$ & $\begin{array}{l}S D S_{2} \text { at } \\
52 \text { weeks }\end{array}$ & $S D S_{\text {gain }}$ & Gain centile \\
\hline $\begin{array}{r}3 \\
2 \\
1 \\
0 \\
-1\end{array}$ & $\begin{array}{r}1 \\
0 \\
-1 \\
-2 \\
-3\end{array}$ & $\begin{array}{l}-0.5 \\
-1.1 \\
-1.7 \\
-2.3 \\
-2.9\end{array}$ & $\begin{array}{c}33 \\
15 \\
5 \\
1 \\
0 \cdot 2\end{array}$ \\
\hline
\end{tabular}

months they have shrunk by a quarter (the third conditional curve down, that started at 2.67 SD scores, crosses the top distance curve at $2 \mathrm{SD}$ scores), and at 2 years by a half (the top conditional curve, starting at $4 \mathrm{SD}$ scores, dips below the $2 \mathrm{SD}$ scores distance curve). This is a graphical demonstration of regression to the mean.

Figures 2 and 3 show the median pattern of weight gain, but they are not useful for identifying growth that departs from this median. For example, how likely is an infant to fall from $\mathrm{SDS}_{1}=+2$ at 4 weeks to the median SD score $=0$ at 52 weeks - is this a sign of growth faltering or just regression to the mean? Equation (1) provides the answer. Substitute $\mathrm{SDS}_{1}=2, \mathrm{SDS}_{2}=0$, and the 4-52 week correlation $\mathrm{r}=0.467$ (table 1), giving the conditional weight gain SD score:

$$
\operatorname{SDS}_{\text {gain }}=\frac{0-0 \cdot 467 \times 2}{\sqrt{1-0 \cdot 467^{2}}}=-1 \cdot 1
$$

equal to the 15 th centile. In other words this growth pattern is not that unusual, and $15 \%$ of Cambridge infants on weight SD score +2 at 4 weeks fall to the median or below at 52 weeks.

Table 2 compares this infant with four others, each of them falling two units of distance SD score between 4 and 52 weeks, starting at $+3,+2,+1,0$, and -1 SD scores respectively. Depending on the starting point, the conditional gain SD score varies between -0.5 and $-2 \cdot 9$, corresponding to conditional gain centiles between 33 and $0 \cdot 2$. A large baby starting at $+3 \mathrm{SD}$ scores is on the 33 rd conditional centile, and so has a one in three chance of falling 2 SD score units or more. Falling to the median (that is from SD score +2 to 0 ) corresponds to the 15 th conditional centile; crossing the median (SD score +1 to -1 ) is on the 5 th conditional centile, and falling from the median (SD score 0 to -2 ) is equivalent to the 1 st conditional centile. For babies starting at -1 SD score, to fall 2 SD score units is very rare - only $2 / 1000$ will falter to this extent. So regression to the mean has a practically important effect, and poor growth cannot be diagnosed from weight change alone - the infant's distance SD score at the start must also be taken into account.

The opposite pattern of growth is to grow along a given centile, and if the centile is sufficiently extreme this is also unusual. For example, to stay on the 2 nd centile from 4 to 52 weeks is equivalent to a conditional gain SD score of $-1 \cdot 2$, equal to the 12 th centile. So for
Table 3 Regression analysis of the transformed correlation between successive weight $S D$ scores, as a function of the time gap between measurements and the infant's mean age (both measured in weeks); dependent variable: Fisher's transformation of correlation

\begin{tabular}{lclrl}
\hline Variable & Coefficient & $S E$ & $\mathrm{t}$ & Probability \\
\hline Constant & 2.98 & 0.13 & 22.5 & $<0.0001$ \\
$\log _{\mathrm{e}}$ gap & -1.67 & 0.058 & -28.6 & $<0.0001$ \\
$\log _{\mathrm{e}}$ age $\times \log _{\mathrm{e}}$ gap & 0.251 & 0.014 & 17.6 & $<0.0001$ \\
$\operatorname{lgap}$ & -2.06 & 0.16 & -12.7 & $<0.0001$ \\
$\log _{\mathrm{e}}$ age & 0.590 & 0.080 & 7.4 & $<0.0001$ \\
$\log _{\mathrm{e}}$ age $)^{2}$ & -0.104 & 0.016 & -6.5 & $<0.0001$ \\
\hline $\mathrm{n}=105 ; R^{2}=99.3 \%$; residual $S D=0.039$ on $99 \mathrm{df}$. &
\end{tabular}

a group of infants on the 2 nd centile at 4 weeks, only $12 \%$ of them are likely to be on or below the 2nd centile at 52 weeks, rather than the $50 \%$ that one might expect.

\section{MODEL CORRELATION}

So long as infants are measured at ages close to those in table 1, equation (1) can be used to calculate conditional weight gain. However, most infants are not measured at these precise ages, and a way is needed to deal with measurements at other times. This is done by interpolating between the tabulated ages, using a regression model of the transformed correlation as a function of the two measurement ages. Table 3 summarises the fitted regression model, which involves logarithmic and inverse transformations of the mean age of measurement and the time gap between measurements.

Put simply, the model shows that the correlation increases as the time gap decreases and the mean age increases. The model fits well, accounting for $99.3 \%$ of the variation in the transformed correlation. Using the formula in table 3 and back transforming, it is possible to calculate the value of $r$ in equation (1) for any pair of measurement ages between 4 weeks and 2 years.

The residual SD of the transformed correlation in table 3 is 0.039 , giving a $95 \%$ confidence interval of $\pm 0 \cdot 08$. This converts back to a $95 \%$ confidence interval of \pm 0.05 for a correlation of $0 \cdot 5, \pm 0.03$ for correlation 0.8 , and \pm 0.007 for correlation 0.95 .

\section{VALIDATION}

Both weight SD score and conditional weight gain SD score $\left(\right.$ SDS $\left._{\text {gain }}\right)$ should have a mean of zero and a SD of 1 . In addition SDS $_{\text {gain }}$ ought to be uncorrelated with the first weight, $\mathrm{SDS}_{1}$. This is tested for in the Cambridge and Newcastle datasets using all possible pairs of weights for each infant, a total of 26264 pairs in Cambridge and 15405 in Newcastle.

To do this, the weight gain SD scores are grouped by mean age and time gap, each split into 16 groups, corresponding roughly to the original 15 measurement ages. The mean age groups are $<8,8+, 12+, 16+, 20+, 24+$, $28+, 32+, 36+, 40+, 44+, 48+, 52+, 65+$, $78+$, and $91+$ weeks, while the time gap groups are $<6,6+, 10+, 14+, 18+, 22+$, $26+, 30+, 34+, 38+, 42+, 46+, 50+, 63+$, $76+$, and $89+$ weeks. This gives 256 possible age gap combinations, of which 108 contain 
Table 4 Pairs of weight SD scores for each infant in Cambridge and Newcastle grouped according to the mean age and time gap between the measurements. For each group the mean and $S D$ of weight gain $S D$ score is calculated, along with the correlation between weight gain SD score and the first weight $S D$ score. The table gives the three quartiles and interquartile range across the groups for the sample size, means, SDs, and correlations. Ideally the means and correlations should be 0 and the $S D$ 1; values are median (lower quartile, upper quartile, interquartile range)

\begin{tabular}{llc}
\hline $\begin{array}{l}\text { Statistic derived } \\
\text { for each group }\end{array}$ & Cambridge $(108$ groups) & Newcastle $(90$ groups $)$ \\
\hline Sample size $_{\text {Mean SDS }}$ & $246(243,249,6)$ & $130(87,182,95)$ \\
SD SDS $_{\text {gain }}$ & $-0 \cdot 12(-0 \cdot 17,-0.07,0 \cdot 10)$ & $0 \cdot 11(0 \cdot 00,0 \cdot 16,0 \cdot 16)$ \\
Correlation $_{\text {(SDS }}$ gain, & $1.00(0.97,1.02,0.05)$ & $1.05(1 \cdot 00,1 \cdot 10,0 \cdot 10)$ \\
SDS $\left._{1}\right)$ & $-0.01(-0.04,0.03,0.07)$ & $-0.09(-0 \cdot 16,0 \cdot 00,0 \cdot 16)$ \\
\hline
\end{tabular}

data in Cambridge, and (excluding those with fewer than 50 points) 90 in Newcastle.

Table 4 summarises the distribution across groups of the mean and SD of SDS gain and its correlation with $\mathrm{SDS}_{1}$. The Cambridge median group size is 246 , slightly greater than the 223 used for the correlations in table 1, while in Newcastle the groups are smaller, median 130. The median across groups for mean SDS $_{\text {gain }}$ in Cambridge is -0.12 , with an interquartile range of $0 \cdot 10$. In Newcastle the median of mean SDS $_{\text {gain }}$ is $0 \cdot 11$, interquartile range $0 \cdot 14$. The greater weight gain of Newcastle infants over the first year as compared to Cambridge has already been noted. ${ }^{17}$

For the SD and the correlation of SDS ${ }_{\text {gain }}$, the Cambridge results are very close to the expected values ( 1 and 0 respectively), and the interquartile ranges are also small. In Newcastle the SD is slightly greater, and the correlation somewhat less than in Cambridge, with much wider interquartile ranges.

The agreement between Newcastle and Cambridge is reasonable on the whole, and it confirms that the conditional reference can be applied to other data.

WEIGHT GAIN CHART

After calculating the conditional weight gain SD score from equation (1), the next question

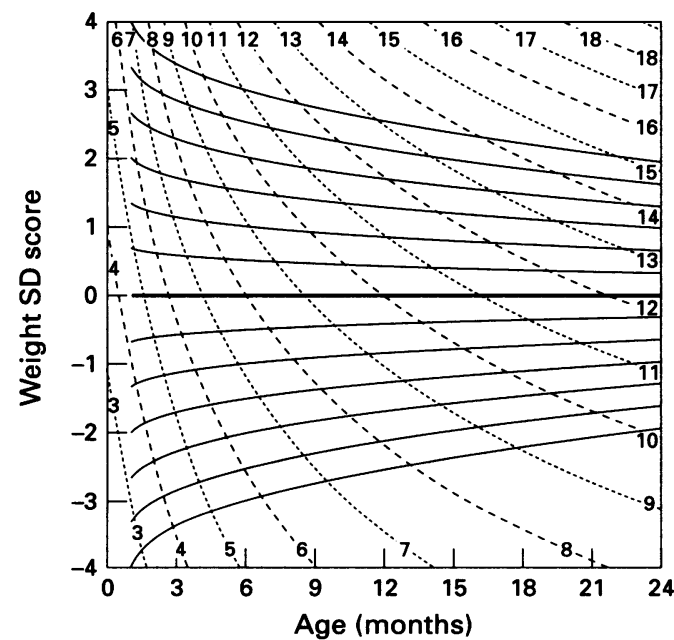

Figure 4 Boys conditional weight chart of fig 2 redrawn with weight SD score on the $Y$ axis. Distance centiles on this scale are horizontal straight lines (not shown), while the conditional centiles shrink towards the median on the $X$ axis. The dashed lines running from top left to bottom right are isoponds, contours of constant weight that allow data to be plotted directly on the chart. See text for details. is how to display it, ideally on the same charts as weight distance SD score. One way to do this is to plot the weight distance chart as weight SD score (rather than weight itself) against age. This converts the distance centiles of figs 2 and 3 to horizontal lines, and the conditional centiles become symmetric curves that shrink towards the median with increasing age, as shown in figs 4 and 5 . Weight can be plotted directly on such a chart using isoponds. ${ }^{18} 19$ Isoponds are contour lines of constant weight which are superimposed on the chart (falling from left to right, shown dotted), and which are easy to derive from the formulas defining the reference centiles (see Appendix).

With weight distance plotted on an SD score, it becomes possible to plot SDS $_{\text {gain }}$ on the same scale, so that weight distance and conditional weight gain appear on the same chart. The weight gain calculated between two ages is shown as a horizontal straight line at the appropriate SD score level, extending between the two ages.

Figure 6 illustrates such a chart with Newcastle subject 2149, measured eight times between 6 weeks and 20 months. Her conditional gain is enormously variable, with SDS $_{\text {gain }}$ in the range $\pm 2 \cdot 5$, that is covering the 1 st to the 99th centile. However, her conditional gain over the whole 18 month period is obviously unremarkable, as her starting and finishing weights are both near the median. This highlights the fact that SDS $_{\text {gain }}$ measured over short periods of time can be very variable due to measurement error.

The advantage of conditional weight gain is that it can be calculated over longer time intervals, to reduce the effect of measurement error. Figure 7 extends fig 6 by showing subject 2149's conditional weight gain calculated for all 28 pairs of measurement occasions. The rising trend from 6 weeks to 5 months emerges as very unusual, with $\mathrm{SDS}_{\text {gain }}=+3$, while both the falling trend between 6 and 9 months $\left(\mathrm{SDS}_{\text {gain }}=-2 \cdot 5\right)$, and the rise from 9 to 12 months $\left(\mathrm{SDS}_{\mathrm{gain}}=+2 \cdot 3\right)$, are also atypical. Despite this, the conditional gain from 6 weeks to 20 months is quite unexceptional, with

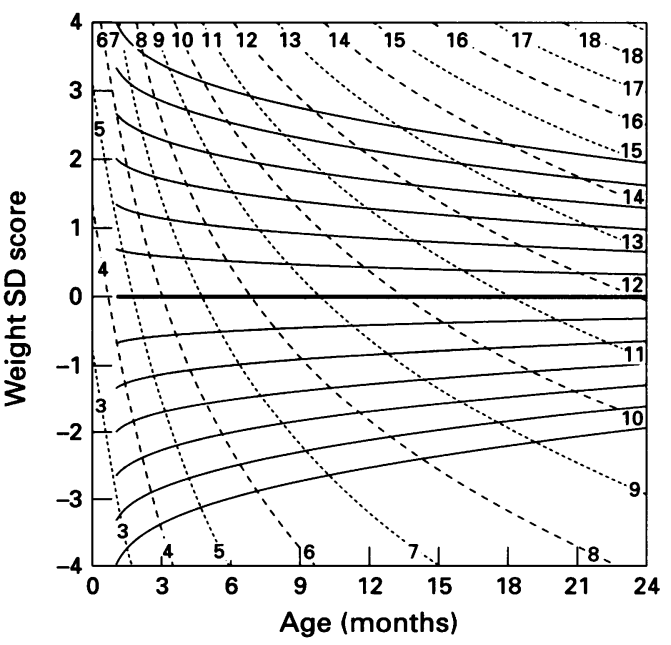

Figure 5 Girls conditional weight chart of fig 3 redrawn with weight $S D$ score on the $Y$ axis. See caption to fig 4 for details. 


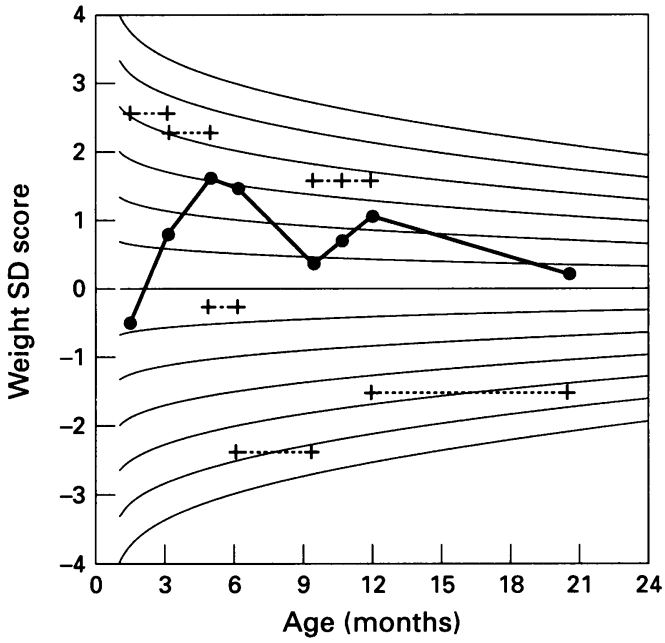

Figure 6 A Newcastle infant's series of weights plotted on the chart of fig 5, with conditional weight gain SD score between successive pairs of measurements displayed as horizontal lines between the two measurement ages. Gain $S D$ score is very variable.

$\operatorname{SDS}_{\text {gain }}=+0 \cdot 5$. There are two warnings here: firstly, weight gain measured over short time periods should be interpreted cautiously, and secondly, normally growing children are likely to throw up the occasional significant weight gain simply by chance, due to the large number of possible measurement pairs.

\section{Discussion}

Monitoring growth during infancy is not as simple as it seems. To express weight gain as a centile or SD score requires knowledge of the mean and $\mathrm{SD}$ of weight gain between arbitrary ages, when published information on this is restricted mainly to time intervals of one, three, or six months. Furthermore, there has until recently been no convenient way of adjusting for regression to the mean.

The solution, as proposed here and elsewhere, ${ }^{47}$ is to convert weight to an SD score using the UK 1990 reference, ${ }^{11}$ and then to use a conditional reference, based on weight correlations from the Cambridge Infant Growth Study, to adjust the change in weight SD score for regression to the mean. This is the phenomenon which causes small infants to grow faster on average than large infants. Fundamentally a statistical phenomenon, as explained earlier, it arises from the natural variation in weight gain from child to child. The variation is a combination of measurement error and variation in the underlying true rate of weight gain, so that both components of variation contribute to regression to the mean.

The mathematical basis for the regression adjustment is simple. For a child measured once and later measured again, weight distance SD score on the second occasion is on average equal to the previous SD score multiplied by the correlation between weight SD score at the two ages. This is always less than 1 , so the expected SD score shrinks towards zero with passing time. In theory the second measurement could be so remote from the first that the correlation is zero, in which case the infant's

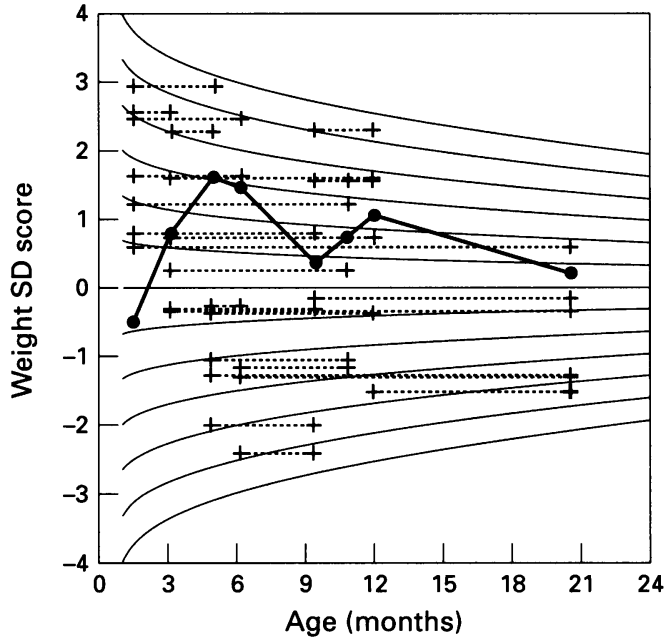

Figure 7 The same chart as fig 6, but with conditional weight gain SD score shown for all pairs of measurements. Gain SD score calculated over long periods of time is less variable than for shorter periods.

expected SD score on the second occasion is also zero, corresponding to median weight.

The thrive index of Wright et al ${ }^{7}$ has much in common with the present paper, based on weight SD score at 12 months adjusted for weight SD score at 6 weeks in Newcastle infants, and given by:

Thrive index $=\mathrm{SDS}_{2}-0 \cdot 62 \times \mathrm{SDS}_{1}-0 \cdot 225$.

The value of 0.62 is the regression coefficient of $\mathrm{SDS}_{2}$ on $\mathrm{SDS}_{1}$, and the constant 0.225 is the average difference in thrive index between the Newcastle infants and the growth reference on which their weight SD score was based. This corresponds to a correlation coefficient of 0.58 , slightly greater than the correlation of 0.54 predicted from the regression model of table 3.

The advantage of the present approach over that of Wright et $a l^{7}$ is that exact rather than approximate ages can be used and weight gain is expressed as an SD score. Another advantage of exact ages is that when there are more than two weights per child, weight gain can be calculated across several time intervals, as in fig 7. Variability in weight from one occasion to the next arises from a combination of measurement error and biological variation in the true rate of weight gain. The longer the time interval the smaller the measurement error contribution, so weight gain should be calculated over the longest available time interval. The form of fig 7, although somewhat cluttered, provides all the relevant information in a single chart.

Another reason why conditional weight gain is so variable (fig 6) is because it is negatively correlated with the previous value. ${ }^{20}$ This means that a high gain is often followed by a low gain, as can happen if one particular weight is discrepant, due either to illness or poor measuring technique. The degree of negative correlation is less over longer time intervals, and it is another reason why weight gain should be derived over as long an interval as possible.

The regression model in table 3 fits the correlation matrix of table 1 well, with over $99 \%$ 
of the variance accounted for. In addition the results of table 4 show that the mean and SD of SDS $_{\text {gain }}$ are close to the expected values in both the Cambridge and Newcastle datasets. This encourages the belief that the conditional gain reference described here is generally valid for British infants.

There are small discrepancies in the distribution of $\mathrm{SDS}_{\text {gain }}$ in table 4, and more so for the Newcastle than the Cambridge data, but in practice the mean and correlation are not critical. The mean of SDS $_{\text {ain }}$ differing from zero indicates only that the group of infants grows at a slightly different rate from the reference, but it does not invalidate the cal-

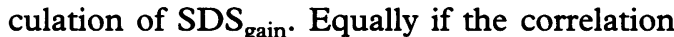
between $\operatorname{SDS}_{\text {gain }}$ and SDS $_{1}$ in table 4 is nonzero, it means that regression to the mean is not completely adjusted for, but again this has only a marginal effect on SDS ${ }_{\text {gain }}$.

The most important aspect of the distribution of SDS $_{\text {gain }}$ is its SD. If the SD is inflated, the distribution is widened and there are a larger than expected number of extreme gains. For example about $2 \cdot 3 \%$ of gains should be below SD score -2 , but if the SD of $\mathrm{SDS}_{\text {gain }}$ were $1 \cdot 1$ rather than 1 , this figure would increase to $3 \cdot 4 \%$ - some $50 \%$ bigger. The message is that unless the $\mathrm{SD}$ is close to 1 , caution is needed when interpreting low values of $\mathrm{SDS}_{\text {gain }}$ - they need to be very low before they are taken too seriously.

The conditional centiles in figs 2-5 are drawn assuming that each child is measured four weekly throughout the first year. However, the measurements could equally have been say every eight, 12, or 24 weeks. Calculating regression to the mean for these various measurement intervals uses different sets of correlations in table 1 . For example the 4 and 52 week measurements have a correlation of 0.47 , as compared with the value of 0.64 obtained by multiplying together all the four week correlations. Equally the correlations for measurements at 4,28 , and 52 weeks estimate regression to the mean from 4 to 52 weeks as 0.51 .

This discrepancy arises because, strictly speaking, weight is better predicted if more than one previous weight is used. It is a small effect, but it highlights the difficulty of constructing a conditional gain chart. If the centiles were based on measurements say eight or 12 weeks apart rather than four, regression to the mean would be greater. The lowest conditional centile curve in fig 2 regresses to the mean from -2 to $-1 \cdot 28$ over the first year, using the factor of 0.64 , while if the direct 4-52 week correlation of 0.47 were used (as it should be), the 52 week SD score would be -0.94 rather than $-1 \cdot 28$, half a centile channel higher. It is not an enormous discrepancy, but it shows that the chart is a compromise. The correct way to adjust for regression to the mean between two ages is to use equation (1), and a computer is better than a chart for this. The value of the chart is that it displays a conservative estimate of regression to the mean.

The approach presented here is specifically targeted at weight during infancy. However, it can be applied equally to height during childhood, ${ }^{21}$ when surveillance for short stature is the main concern. The principle of extending the time period leads naturally to the concept of conditional height gain measured over two or three years rather than the usual 1 , for example, between the toddler stage and school entry.

In the future there will be specialist software which will do the calculations and draw the chart on the computer screen - all that is needed is a suitable spreadsheet macro. The conditional gain SD score associated with each pair of measurements could be displayed by colour coding the line joining them. A useful start in this respect is the Castlemead growth program, ${ }^{22}$ which converts measurements to SD scores using a suitable reference, and then plots the SD score against age. However, it would need modifying to calculate and/or display the gain SD score.

In conclusion, the conditional gain reference described here allows weight gain between any pair of ages during infancy to be expressed as a conditional gain SD score, which at last makes infant growth monitoring feasible.

I am very grateful to Roger Whitehead, Alison Paul, and Anne Ahmed for their input to the Cambridge Infant Growth Study; Ahm Charlotte Wright for providing me with the Newcastle data, and support; the two anonymous referees for their valuable suggessupport; the two anonymous referees for their valuable sugg
tions, and John Matthews for giving me the original idea.

1 Thompson H. Data on the growth of children during the first year after birth. Hum Biol 1951; 23: 75-92.

2 Boryslawski K. Structure of monthly increments of length, weight and head circumference in the first year: a pure longitudinal study of 200 Wroclaw infants. Ann Hum Biol 1988; 15: 205-12.

3 Healy MJR, Yang M, Tanner JM, Zumrawi FY. The use of short-term increments in length to monitor growth in infancy. In: Waterlow JC, ed. Linear growth retardation in less developed countries. New York: Raven, 1988: 41-55.

4 Cole TJ. The use and construction of anthropometric growth reference standards. Nutrition Research Reviews 1993; 6: $19-50$.

5 Lampl M, Veldhuis JD, Johnson ML. Saltation or stasis: a model of human growth. Science 1992; 258: 801-3.

6 Emery JL, Waite AJ, Carpenter RG, Limerick SR, Blake D. Apnoea monitors compared with weighing scales for siblings after cot death. Arch Dis Child 1985; 60: 1055-60.

7 Wright CM, Matthews JNS, Waterston A, Aynsley-Green A. What is a normal rate of weight gain in infancy? Acta Paediatr 1994; 83: 351-6.

8 Healy MJR. Notes on the statistics of growth standards. Ann Hum Biol 1974; 1: 41-6.

9 Cameron N. Conditional standards for growth in height of British children from 5.0 to 15.99 years of age. Ann Hum Biol 1980; 7: 331-7.

10 Berkey CS, Reed RB, Valadian I. Longitudinal growth standards for preschool children. Ann Hum Biol 1983; 10: $57-67$.

11 Freeman JV, Cole TJ, Chinn S, Jones PRM, White EM, Preece MA. Cross sectional stature and weight reference
curves for the UK, 1990. Arch Dis Child 1995; 73: 17-24.

12 Whitehead RG, Paul AA, Cole TJ. Diet and the growth of healthy infants. Fournal of Human Nutrition and Dietetics 1989; 2: 73-84.

13 Heimendinger J, Laird N. Growth changes: measuring the effect of an intervention. Evaluation Review 1983; 7 80-95.

14 Kendall MG, Stuart A. The advanced theory of statistics. 3rd Ed. Vol 2. London: Griffin, 1973.

15 Royston P, Altman DG. Regression using fractional polynomials of continuous covariates: parsimonious parametric modelling (with discussion). Applied Statistics fournal of the Royal Statistical Society Series C 1994; 43: 429-67.

16 Cole TJ. Do growth chart centiles need a facelift? $B M \mathcal{F}$ 1994; 308: 641-2.

17 Wright CM, Waterston A, Aynsley-Green A. Comparison of the use of Tanner and Whitehouse, NCHS, and Cambridge standards in infancy. Arch Dis Child 1993; 69:

18 deWinter ER. Measuring weight gains relative to standard weights-for-age. Environmental Child Health 1977; 23: weights

19 Sorva R, Perheentupa J, Tolppanen E-M. A novel forma for a growth chart. Acta Paediatr Scand 1984; 73: 527-9. 
20 Cole TJ. Relating growth rate to environmental factors methodological problems in the study of growth-infectio interaction. Acta Paediatr Scand 1989; 350 (suppl): 14-20. 21 Cole TJ. Growth charts for both cross-sectional and longitudinal data. Stat Med 1994; 13: 2477-92.

22 Castlemead Publications. Growth program: software for growth analysis. Welwyn Garden City: Castlemead Publications, 1993.

23 Cole TJ, Green PJ. Smoothing reference centile curves: the LMS method and penalized likelihood. Stat Med 1992; 11: 1305-19.

\section{Appendix}

To convert an infant's weight to a SD score based on the UK reference, ${ }^{11}$ quantities $L, M$, and $S$ for the infant's age and sex are required. Substitute L, $M$, and $S$ into the formula ${ }^{23}$ :

$$
\text { Weight SD score }=\frac{(\text { Weight } / M)^{\mathrm{L}}-1}{\mathrm{~L} \times \mathrm{S}} .
$$

To draw each isopond in figs 4 and 5 , weight in formula (A1) is set to the required value, and appropriate values of $L, M$, and $S$ for a series of ages are also substituted. This resulting series of SD score values plotted against age gives the isopond.

L, $M$, and $S$ values for weight can be obtained on diskette through the Child Growth Foundation, 2 Mayfield Avenue, London W4 1PW. 